\title{
Fatores Associados à Qualidade de Vida Relacionada à Saúde de Pacientes com Câncer em Tratamento Clínico
}

doi: https://doi.org/10.32635/2176-9745.RBC.2019v65n2.395

\author{
Factors Associated with Health-Related Quality of Life of Cancer Patients Undergoing Clinical Treatment \\ Factores Asociados con la Calidad de Vida Relacionada con la Salud de los Pacientes con cáncer en Tratamiento Clínico
}

Aline Saltaréllo de Jesus ${ }^{1}$; Simara Rodrigues Ajala²; Camila Armstrong Saldanha; Maria Cláudia Bernardes Spexoto ${ }^{4}$

Resumo

Introduçáo: Estado nutricional, qualidade de vida relacionada à saúde (QVRS) e apetite preservados proporcionam maior sobrevida aos pacientes oncológicos. Suas oscilaçóes são comuns durante o tratamento, mas pouco se conhece sobre os fatores interferentes na QVRS. Objetivo: Avaliar os fatores associados à QVRS de pacientes com câncer. Método: Estudo transversal com 110 pacientes com câncer em tratamento clínico. Foram coletadas as variáveis sociodemográficas, clínicas, QVRS, estado nutricional e apetite. Os instrumentos utilizados foram o European Organization for Research and Treatment of Cancer Quality of Life Questionnaire Core 30, a Avaliação Subjetiva Global (ASG) e o Cancer Appetite and Symptom Questionnaire. Para análise estatística, considerou-se o nível de significância de 5\%. Resultados: A maioria era do sexo feminino (62,72\%) com média de idade igual a 74,41 $\pm 24,54$ anos, casada (70,0\%), sem atividade de trabalho $(51,82 \%)$ e pertencente à classe econômica B $(54,54 \%)$. O tipo de câncer mais prevalente nessa população foi o câncer de mama (30,0\%), com estadiamento IV $(34,44 \%)$ e ausência de metástases $(53,64 \%)$. O comprometimento do apetite foi identificado como fator associado à QVRS, apresentando diferença significativa na saúde global e na $\mathrm{QV}(\mathrm{p}<0,001)$. A função emocional foi a mais prejudicada. A fadiga foi o sintoma que apresentou maior pontuaçáo. Conclusáo: $\mathrm{O}$ comprometimento do apetite apresentou associação com a saúde global e a QV dos pacientes em tratamento oncológico.

Palavras-chave: Neoplasias; Qualidade de Vida; Apetite; Estado Nutricional.

\begin{abstract}
Introduction: Protected nutritional status, health-related quality of life (HRQOL) and appetite provide longer survival for cancer patients. Oscillations are common during treatment, but little is known about factors interfering with HRQOL. Objective: To assess the factors associated with the HRQOL of patients with cancer. Method: A cross-sectional study with 110 cancer patients undergoing clinical treatment. Sociodemographic, clinical, HRQOL, nutritional status and appetite variables were collected. The instruments used were the European Organization for Research and Treatment of Cancer Quality of Life Questionnaire Core 30, the Subjective Global Assessment (SGA) and the Cancer Appetite and Symptom Questionnaire. For statistical analysis, the level of significance was $5 \%$. Results: The majority were female $(62.72 \%)$ with a mean age of $74.41 \pm 24.54$ years, married $(70.0 \%)$, without working activity $(51.82 \%)$ and belonging to economic class B (54.54\%). The most prevalent type of cancer in this population was breast cancer $(30.0 \%)$, with staging IV (34.44\%) and absence of metastasis (53.64\%). The appetite impairment was identified as a factor associated with HRQOL, presenting significant difference in the global health and QoL (quality of life). Emotional function was the most damaged. Fatigue was the symptom that presented the highest score. Conclusion: Appetite impairment was associated with the global health and QoL of cancer patients in oncologic treatment.

Key words: Neoplasms; Quality of Life; Appetite; Nutritional Status.
\end{abstract}

Resumen

Introducción: Estado nutricional, calidad de vida relacionada con la salud (CVRS) y apetito conservado proporcionan una supervivencia más larga para los pacientes con cáncer. Sus oscilaciones son comunes durante el tratamiento, pero se sabe poco sobre los factores de interferencia en la CVRS. Objetivo: Evaluar los factores asociados con la CVRS de los pacientes con cáncer. Método: estudio transversal con 110 pacientes con cáncer en tratamiento clínico. Incluimos variables sociodemográficas, clínicas, de CVRS, estado nutricional y apetito. Los instrumentos utilizados fueron el Cuestionario básico 30 de la Organización Europea para la Investigación y el Tratamiento del Cáncer, la Evaluación global subjetiva (EGS) y el cuestionario sobre el apetito y los síntomas del cáncer. Se consideró nivel de significancia del 5\%. Resultados: La mayoría era mujeres (62,72\%) con una edad media de $74,41 \pm 24,54$ años, casada $(70,0 \%)$, sin actividad laboral $(51,82 \%)$ y perteneciente a clase económica B $(54,54 \%)$. El tipo de cáncer más frecuente era el cáncer de mama $(30,0 \%)$, estadificación IV $(34,44 \%)$ y sin metástasis $(53,64 \%)$. El deterioro del apetito fue identificado como factor asociado con la CVRS, mostrando diferencia significativa en lo generale de salud y CV. La función emocional fue la más deteriorada. Fatiga fue el síntoma con el puntaje más alto. Conclusión: El deterioro del apetito se asoció con la salud general y CV de los pacientes en tratamiento contra el cáncer.

Palabras clave: Neoplasias; Calidad de Vida; Apetito; Estado Nutricional.

\footnotetext{
1 Universidade Federal da Grande Dourados (UFGD). Dourados (MS), Brasil. Orcid iD: https://orcid.org/0000-0002-7196-470X

2 UFGD. Dourados (MS), Brasil. Orcid iD: https://orcid.org/0000-0001-6164-1272

${ }^{3}$ Oncoclínica Dourados. Dourados (MS), Brasil. Orcid iD: https://orcid.org/0000-0002-4671-2228

${ }^{4}$ UFGD. Dourados (MS), Brasil. Orcid iD: https://orcid.org/0000-0001-7681-1422

Endereço para correspondência: Maria Cláudia Bernardes Spexoto. UFGD/Faculdade de Ciências da Saúde/Curso de Nutrição. Rodovia Dourados, Itahum, Km 12 - Unidade II - Cidade Universitária - Caixa Postal: 364. CEP 79.804-970. Dourados (MS), Brasil. E-mail: mariaspexoto@ufgd.edu.br
} 


\section{INTRODUÇÃO}

O câncer é a segunda principal causa de morte no mundo e foi responsável por 9,6 milhóes de mortes no ano de 2018. Em nível global, uma em cada seis mortes está relacionada a essa doença. De acordo com a Organizaçâo Mundial da Saúde (OMS), aproximadamente 70\% das mortes por câncer ocorrem em países de baixa e média rendas ${ }^{1}$, sendo um dos principais problemas de saúde pública no Brasil. Segundo o Instituto Nacional de Câncer José Alencar Gomes da Silva (INCA) ${ }^{2}$, a estimativa para cada ano do biênio 2018-2019 no Brasil é de mais de 600 mil casos novos de câncer, sendo as neoplasias de próstata (68 mil) mais frequentes nos homens e mama (60 mil) nas mulheres.

Com os avanços nos tratamentos, a sobrevida dos pacientes com câncer tem aumentado ao longo dos anos. Dessa forma, surgiu um grande interesse por parte dos pesquisadores em investigar a qualidade de vida relacionada à saúde (QVRS) e os impactos do tratamento ${ }^{3}$.

A QVRS se refere às percepçôes subjetivas dos aspectos positivos e negativos dos sintomas dos pacientes com câncer, incluindo funçóes físicas, emocionais, sociais e cognitivas e, principalmente, sintomas de doenças e efeitos colaterais do tratamento ${ }^{4}$.

São inúmeras as repercussôes negativas e vários os sintomas de impacto nutricional advindos do tratamento e/ou da própria doença 5 . Entre estes, podem ser citados os distúrbios no apetite, sendo a anorexia primária (no âmbito do sistema nervoso central) a causa mais comum da falta de apetite e da redução da ingestão de alimentos, especialmente àqueles submetidos à quimioterapia ${ }^{6}$. A falta de apetite pode ser atribuída também às alteraçôes de sabor e cheiro dos alimentos, no paladar, à saciedade precoce, bem como a náuseas e vômitos, entre outros ${ }^{7}$.

$\mathrm{Na}$ prática clínica, é indispensável a utilização de instrumentos que investiguem de forma precoce o aparecimento desses sintomas, pois estes podem levar o paciente à piora do curso da doença, à menor adesão ao tratamento e ao aumento da morbimortalidade, o que justifica a importância de se investigar também o estado nutricional, a QVRS e o apetite, pois sabe-se que pacientes com o estado nutricional preservado, boa QVRS e baixo comprometimento do apetite apresentam maior sobrevida e o peso corporal geralmente mais saudável.

São conhecidos na literatura os efeitos colaterais do tratamento, da QVRS e do estado nutricional dos pacientes com câncer, mas pouco se investiga sobre esses aspectos na QVRS, especialmente sobre as alteraçóes do apetite, com a utilização de instrumentos específicos e validados para análise do estado nutricional, da QVRS e do comprometimento do apetite. Dessa forma, este estudo foi realizado com o objetivo de avaliar os fatores associados à QVRS de pacientes com câncer.

\section{MÉTODO}

Foi conduzido um estudo transversal, com delineamento amostral não probabilístico por conveniência. Participaram 110 pacientes atendidos em uma clínica privada no interior do Mato Grosso do Sul, Regiáo Centro-Oeste do Brasil. O período de coleta de dados foi compreendido entre os meses de novembro de 2017 e maio de 2019.

Foram incluídos pacientes adultos, de ambos os sexos, com diagnóstico de neoplasia maligna em tratamento clínico que concordaram e assinaram o Termo de Consentimento Livre e Esclarecido (TCLE).

Foram adotados como critérios de exclusão pacientes submetidos a procedimentos cirúrgicos de grande porte, com características que impossibilitassem o preenchimento dos questionários do estudo e antropometria, como déficit cognitivo ou disfunções psiquiátricas graves, indivíduos com idade inferior a 20 anos, gestantes, puérperas, lactantes e população indígena.

Para a caracterização amostral, foram consideradas variáveis sociodemográficas e clínicas. A sociodemográficas incluíram grupo etário (adulto/idoso), sexo, idade, estado civil, presença de atividade laboral e classe econômica. A idade foi analisada em anos completos, a presença de atividade laboral foi avaliada de forma dicotômica (presença/ausência). A classe econômica foi determinada a partir do cômputo proposto pelo Critério de Classificação Econômica Brasil da Associação Brasileira de Empresas de Pesquisa ${ }^{8}$.

Entre as variáveis clínicas, foram coletados: o tipo de tratamento, grupos diagnósticos, estadiamento clínico da doença e metástase (presença/ausência).

Para estabelecer os grupos diagnósticos, inicialmente foram coletados os diagnósticos primários dos pacientes e, em seguida, estes foram agrupados segundo sistemas do corpo humano.

Cabe esclarecer que as variáveis sociodemográficas foram obtidas em forma de entrevista face a face e as variáveis clínicas, por consulta ao prontuário eletrônico do paciente.

Para avaliar a QVRS, foi utilizado o instrumento European Organization for Research and Treatment of Cancer Quality of Life Questionnaire Core 30 (EORTC/ QLQ-C30), em sua terceira versão, na língua portuguesa, proposto pelo European Organization for Research and Treatment of Cancer ${ }^{4}$. Esse instrumento é composto por 30 itens, com escala de resposta do tipo Likert disposta em quatro pontos (não $=1$, pouco $=2, \operatorname{moderado}=3 \mathrm{e}$ muito=4), com exceção da escala de saúde global e 
QV (itens 29 e 30) que se dá entre 1 a 7 (1=péssimo e $7=$ excelente). Os itens são subdivididos em cinco escalas funcionais: 1) função física $(\mathrm{FF}), 2$ ) função cognitiva (FC), 3) função emocional (FE), 4) função social (FS) e 5) desempenho de papel (DP); três escalas de sintomas: fadiga, dor, náuseas e vômito; cinco itens isolados que também avaliam sintomas: dispneia, apetite, insônia, constipação e diarreia; um item que avalia dificuldade financeira (DF) do tratamento da doença e uma escala geral de saúde global e QV.

$\mathrm{O}$ instrumento possui pontuação entre 0 e 100, onde uma pontuaçáo mais alta representa uma escala de resposta mais alta. Com relação à sua interpretação, uma pontuação mais alta para as escalas funcionais representa um nível alto/ saudável de função. Entretanto, nas escalas de sintomas e itens isolados, quanto mais elevada a pontuação, maior comprometimento/presença de sintomas (alto nível de sintomatologia/problemas). Para a escala de saúde global e QV, dar-se-á a mesma interpretação das escalas de função, quanto mais elevada a pontuação, melhor o estado de saúde geral e a QVRS do paciente ${ }^{4}$.

Para avaliaçáo do acometimento do apetite e presença sintomas de impacto nutricional, foi utilizado o instrumento denominado Cancer Appetite and Symptom Questionnaire (CASQ), proposto inicialmente na língua inglesa por Halliday et al. ${ }^{9}$ No presente estudo, foi utilizada a versão na língua portuguesa ${ }^{10}$, composta por dez itens com respostas dispostas em escala do tipo de Likert de cinco pontos, exceto o item 12 (referente à "dor") que possui seis pontos de resposta. Cabe esclarecer que o instrumento possui quatro itens com escala de resposta invertida e que a versão em português não utiliza os itens cinco e seis do instrumento original.

O cômputo do escore global de apetite e sintomas foi efetuado utilizando a equação proposta pelos mesmos autores ${ }^{10}$, conforme a Figura 1, sendo os pacientes categorizados em "baixo comprometimento do apetite" (escore $\leq 1$ ), "moderado comprometimento do apetite" (escore 1-3) e "severo comprometimento do apetite" (escore $>3)$.

Escore global de apetite

$=0,171$ it $1+0,125 i t 2+0,026 i t 3+0,138$ it $4+0,153 i t 7$ $+0,084 i t 8+0,117 i t 9+0,047$ it $10+0,101 i t 11+0,053 i t 12$

Figura 1. Equação do cômputo do escore global de apetite Legenda: it: item

Para avaliação do estado nutricional, foi utilizada a Avaliação Subjetiva Global (ASG) proposta por Ottery ${ }^{11}$ e adaptada culturalmente para o português por Campos et al. ${ }^{12}$. Para a classificação do estado nutricional, adotou-se a proposta original de Ottery ${ }^{11}$, sendo os indivíduos categorizados em "bem nutrido (A)", "moderadamente desnutrido ou suspeita de desnutriçáo (B)" e "gravemente desnutrido (C)".

A fim de complementar essa avaliação, foram consideradas as medidas antropométricas peso $(\mathrm{kg})$ e altura $(\mathrm{cm})$, para posterior cálculo do índice de massa corporal (IMC) $\left(\mathrm{kg} / \mathrm{m}^{2}\right)$. Os pacientes adultos foram classificados de acordo com os pontos de corte estabelecidos pela World Health Organization ${ }^{13}$ e, para os idosos, foram considerados os pontos de corte proposto por Lipschitz ${ }^{14}$.

A análise de dados foi realizada com o auxílio do programa International Business Machines Corporation - Statistical Package for the Social Sciences (IBM SPSS Statistics) (v.22, SPSS An IMB Company, Chicago, IL). Inicialmente, realizou-se análise descritiva dos dados. Para o estudo de comparaçáo dos escores médios de saúde global e QVRS entre as variáveis sociodemográficas, clínicas, estado nutricional e comprometimento do apetite, utilizou-se o teste $t$ de Student para comparação de médias entre variáveis independentes quando havia duas categorias, e a análise de variância (Anova) para comparação de médias, quando existiam variáveis com três ou mais categorias.

Realizou-se análise de regressão linear univariada e múltipla para verificar se as variáveis sociodemográficas, clínicas, do estado nutricional e comprometimento do apetite foram capazes de prever a QVRS. As escalas (domínios) do instrumento EORTC/QLQ-C30 foram consideradas como variáveis dependentes no modelo de regressão. $\mathrm{O}$ desfecho principal foi a variável dependente saúde global e QV (itens 29 e 30 do instrumento).

Para todas as análises, foi considerado nível de significância de $5 \%(\mathrm{p}<0,05)$.

Esta pesquisa obteve parecer favorável do Comitê de Ética em Pesquisa em Seres Humanos sob o número CAAE: 73621317.7.0000.5160 e de parecer: 2.287.209.

\section{RESULTADOS}

Participaram deste estudo 110 pacientes oncológicos em tratamento clínico, sendo 53 adultos com média de idade igual a 47,3 anos (desvio-padrão=9,39) e 57 idosos com média de idade de 71,10 anos (desvio-padrão=8,49). A maioria era do sexo feminino $(62,72 \%)$, casada $(70,0 \%)$, sem atividade de trabalho $(51,82 \%)$ e pertencente à classe econômica $\mathrm{B}$ (54,54\%). O tipo de câncer mais prevalente nessa população foi o câncer de mama $(30,0 \%)$, com estadiamento IV (34,44\%) e ausência de metástases (53,64\%).

O escore médio do comprometimento do apetite dos pacientes foi de $1,29 \pm 0,64$, sendo classificados com moderado comprometimento do apetite.

$\mathrm{Na}$ Tabela 1, foi apresentada a comparação das médias de saúde global e QV dos pacientes segundo as variáveis investigadas. 
Tabela 1. Comparação das médias de saúde global e QV segundo as variáveis sociodemográficas, clínicas, estado nutricional e comprometimento do apetite

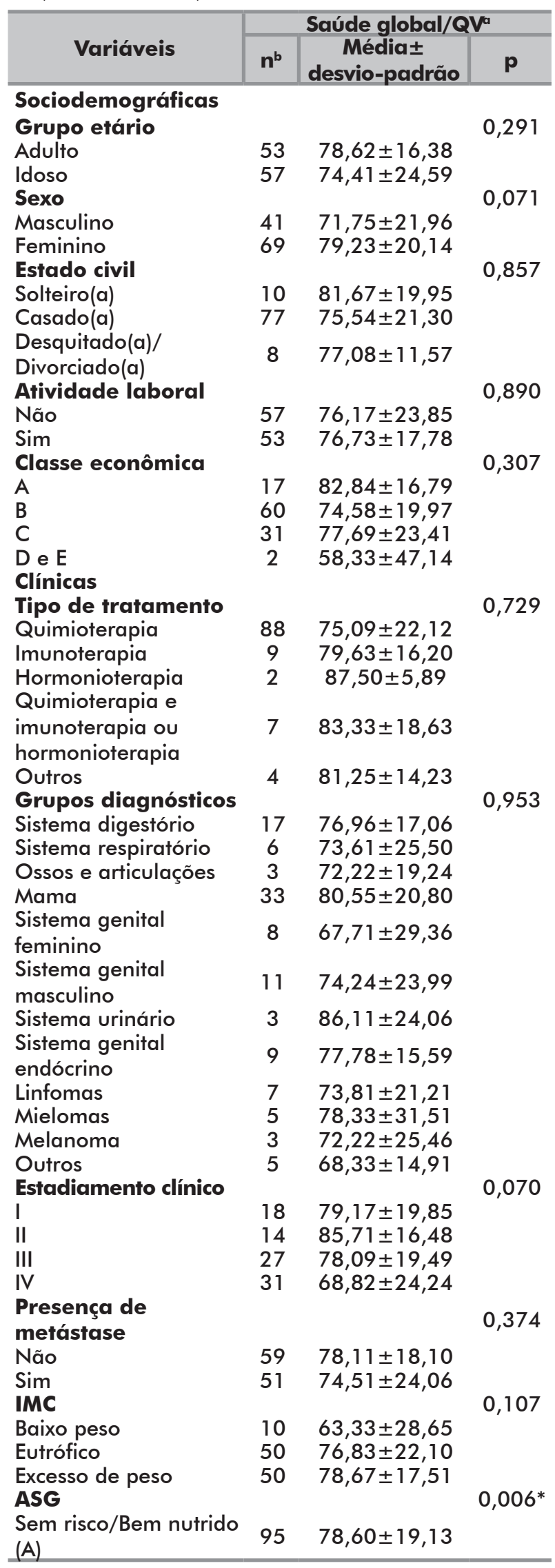

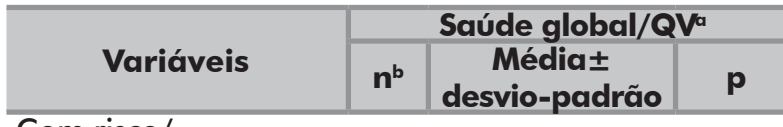

Com risco/

moderadamente

ou gravemente

$15 \quad 62,78 \pm 27,61$

desnutrido (B e C)

Comprometimento

do apetite

$0,008^{*}$

Baixo

comprometimento

Moderado/severo

comprometimento

\author{
$4183,33 \pm 16,35$ \\ $6972,34 \pm 22,53$
}

Legendas: QV: qualidade de vida; IMC: índice de massa corporal; ASG: avaliaçáo subjetiva global.

Notas: aSaúde global/QV: foram utilizados os itens 29 e 30 do instrumento EORTC/QLQ C30. ${ }^{*} \mathrm{p}<0,05$ teste T ou Anova; balgumas informaçóes nấo foram encontradas nos prontuários e/ou náo respondidas pelos pacientes (missing).

Quando avaliadas a saúde global e a QV, segundo as variáveis sociodemográficas e clínicas, observou-se que não houve diferença estatística entre os grupos. Ao comparar as médias de saúde global e QV segundo as variáveis que compuseram o estado nutricional determinado pela ASG, notou-se que os pacientes apresentaram diferença significativa entre as médias; pacientes categorizados com risco nutricional $\mathrm{B}$ e $\mathrm{C}$ apresentaram média inferior $(62,78 \pm 27,61 ; \mathrm{p}=0,006)$ aos pacientes sem risco nutricional (A) $(78,60 \pm 19,13)$. Esse resultado sugere que os pacientes que apresentam algum grau de desnutrição possuem QVRS menos elevada.

Observou-se ainda que a saúde global/QV dos pacientes com comprometimento moderado ou grave do apetite foi menor e estatisticamente significativa $(72,34 \pm 22,53 ; \mathrm{p}=0,008)$ quando comparada aos pacientes que apresentaram baixo comprometimento do apetite $(83,33 \pm 16,35)$. Dessa forma, pode-se afirmar que, para a amostra estudada, a saúde global/QV está relacionada com o quáo comprometido está o apetite do paciente e a presença de outros sintomas de impacto nutricional.

A maioria dos pacientes referiu não ter dificuldade alguma ao fazer uma curta caminhada fora de casa $(74,5 \%)$, não precisar ficar na cama ou em uma cadeira durante o dia $(66,1 \%)$, não precisar de ajuda para se alimentar, se vestir, se lavar ou usar o banheiro $(94,5 \%)$, referiu não apresentar limitaçóes para realizar seu trabalho ou fazer outras atividades diárias $(67,1 \%)$, bem como para realizar atividades de divertimento ou lazer $(69,1 \%)$. Para a maioria dos demais itens, os quais correspondem às escalas funcionais, a resposta dos participantes foi "não".

Com relação aos sintomas investigados pelo EORTC/ QLQ-C30, a fadiga, independentemente do grau, foi relatada por $50,5 \%$ dos pacientes entrevistados.

Quando avaliada a saúde em geral, durante a última semana, $32,7 \%(\mathrm{n}=36)$ dos entrevistados avaliaram sua saúde como excelente e, quando questionados sobre a QV 
geral, no mesmo período de uma semana, 30,3\% ( $\mathrm{n}=33)$ também afirmaram sentir-se em condiçôes de excelência.

$\mathrm{Na}$ Tabela 2, estão apresentados as medidas de resumo das escalas funcionais, sintomas, escala global de saúde e QV, e itens isolados de sintomas e dificuldade financeira.

Tabela 2. Medidas de resumo das escalas funcional, sintomas, escala global de saúde e QV e itens isolados de sintomas e impacto financeiro do instrumento EORTC/QLQ C-30

\begin{tabular}{|c|c|c|c|}
\hline \multirow{2}{*}{$\begin{array}{c}\text { Escalas } \\
\text { Escalas } \\
\text { funcionais }\end{array}$} & \multirow{2}{*}{$\begin{array}{c}\text { Média } \\
\text { desvio-padrão }\end{array}$} & \multirow[b]{2}{*}{ Mínimo } & \multirow[b]{2}{*}{ Máximo } \\
\hline & & & \\
\hline FF & $73,87 \pm 26,62$ & 0,00 & 100,00 \\
\hline DP & $76,66 \pm 35,13$ & 0,00 & 100,00 \\
\hline FE & $72,65 \pm 27,53$ & 0,00 & 100,00 \\
\hline $\mathrm{FC}$ & $76,06 \pm 27,69$ & 0,00 & 100,00 \\
\hline FS & $74,24 \pm 29,66$ & 0,00 & 100,00 \\
\hline \multicolumn{4}{|l|}{$\begin{array}{l}\text { Escala de } \\
\text { sintomas }\end{array}$} \\
\hline Fadiga & $30,05 \pm 31,94$ & 0,00 & 100,00 \\
\hline $\begin{array}{l}\text { Náuseas e } \\
\text { vômito }\end{array}$ & $10,00 \pm 20,71$ & 0,00 & 100,00 \\
\hline Dor & $16,66 \pm 29,52$ & 0,00 & 100,00 \\
\hline Dispneia & $9,39 \pm 22,18$ & 0,00 & 100,00 \\
\hline Insônia & $28,48 \pm 37,73$ & 0,00 & 100,00 \\
\hline Apetite & $26,36 \pm 40,15$ & 0,00 & 100,00 \\
\hline Constipação & $20,30 \pm 34,11$ & 0,00 & 100,00 \\
\hline Diarreia & $14,84 \pm 30,48$ & 0,00 & 100,00 \\
\hline DF & $83,33 \pm 32,48$ & 0,00 & 100,00 \\
\hline $\begin{array}{l}\text { Escala global } \\
\text { de saúde/QV }\end{array}$ & $76,44 \pm 21,05$ & 0,00 & 100,00 \\
\hline
\end{tabular}

Legendas: FF: funçáo física; DP: desempenho de papel; FE: função emocional, FC: funçâo cognitiva; FS: funçáo social; DF: dificuldade financeira; QV: qualidade de vida.

É possível observar que, entre as escalas funcionais, o DP apresentou o escore médio mais elevado $(76,66 \pm 35,13)$; ou seja, mais próximo de 100 , sendo, portanto, a função menos prejudicada dos pacientes deste estudo. Em contrapartida, a FE foi a mais comprometida entre os pacientes com escore médio igual a $72,85 \pm 27,53$, uma vez investigados sobre tensão, preocupação, irritabilidade e depressão.

Para as escalas de sintomas, o escore mais elevado foi evidenciado na escala de fadiga $(30,05 \pm 31,94)$. Quanto aos itens isolados, a insônia foi a condição predominante, com escore médio igual a $28,48 \pm 37,73$ e, em seguida, a falta de apetite $(26,36 \pm 40,15)$.

Quando avaliado o item DF, observou-se que este apresenta escore elevado $(83,33)$, demonstrando que a população estudada apresenta dificuldades financeiras provocadas pela condição física e pelo tratamento.

Com relação à escala global de saúde/QV, o escore médio foi de $76,44 \pm 21,05$, estando os pacientes com os aspectos inerentes à $\mathrm{QV}$ preservados.
$\mathrm{Na}$ Tabela 3, encontram-se os fatores associados à QVRS dos pacientes deste estudo.

Nota-se que o comprometimento do apetite apresentou associação significativa, no nível de $\mathrm{p}<0,001$, com as escalas: FF, FE e FC.

O comprometimento do apetite também apresentou relação significativa com as escalas de sintomas e itens isolados: fadiga $(\mathrm{p}<0,001)$, náuseas/vômito $(\mathrm{p}=0,003)$, dor $(\mathrm{p}=0,001)$, dispneia $(\mathrm{p}=0,002)$, apetite $(<0,001)$ e dificuldade financeira $(0,049)$. Além disso, o comprometimento do apetite apresentou impacto na saúde geral e QV dos pacientes $(\mathrm{p}<0,001)$, sendo a única variável independente no modelo com associação significativa nesse domínio, resultando em um modelo que explicou 43,6\% da saúde global e QV percebidas pelo paciente.

Pode-se destacar outras variáveis independentes que apresentaram associação significativa no modelo de regressão, tais como o IMC na $\mathrm{FE}(\mathrm{p}<0,001)$ e sintoma de constipação ( $\mathrm{p}=0,019)$, grupos diagnósticos e ASG na FE $(0,004 ; 0,013$, respectivamente), a ASG na FC $(0,026)$ e sintoma náuseas/vômito $(\mathrm{p}=0,001)$ e atividade laboral no sintoma diarreia $(\mathrm{p}=0,029)$.

\section{DISCUSSÃO}

Este estudo verificou que a maioria dos pacientes entrevistados, mesmo encontrando-se bem nutridos, apresentou moderado ou severo comprometimento do apetite durante o tratamento clínico do câncer e isso deve ser considerado na avaliação da QVRS. A maior contribuição deste estudo foi identificar e reforçar, na literatura da área, que o comprometimento do apetite foi um fator associado à saúde global/QV dos pacientes com câncer em tratamento clínico e, portanto, deve ser identificado precocemente e acompanhado durante todo o curso de tratamento. Neste estudo, o estado nutricional apresentou associação significativa com as escalas FE, FC e náuseas/vômitos, as quais são aspectos inerentes à QVRS do paciente em tratamento do câncer, sendo maioria investigada pela ASG. Salienta-se que o estado nutricional deve ser avaliado, preferencialmente, pela ferramenta ASG ou ASG-PPP (produzida pelo paciente) ${ }^{15,16}$, pois, quando estimado pelo IMC, este não apresentou impacto sobre a saúde global e a QV dos pacientes.

Cabe ressaltar que a Sociedade Brasileira de Nutrição Parenteral e Enteral (Brazilian Society of Parenteral and Enteral Nutrition ${ }^{16}$ recomenda que a ASG ou ASG-PPP seja inserida na prática clínica do atendimento nutricional de pacientes com câncer, por ser um instrumento sensível para avaliar o estado nutricional e diagnosticar casos de risco nutricional. Além disso, essa ferramenta viabiliza o 
Tabela 3. Fatores associados à QV relacionada à saúde dos participantes

\begin{tabular}{|c|c|c|c|c|c|c|c|c|c|c|}
\hline \multicolumn{11}{|c|}{ EORTC/QLQ C-30 (variável dependente) } \\
\hline \multicolumn{11}{|c|}{ Escalas funcionais } \\
\hline \multirow[t]{2}{*}{ Variáveis independentes } & \multicolumn{2}{|c|}{$\begin{array}{c}\mathbf{F F} \\
\mathrm{R}^{2}=0,423 ; \\
\mathrm{R}^{2} \mathrm{aj}=0,315\end{array}$} & \multicolumn{2}{|c|}{$\begin{array}{c}\text { DP } \\
\mathrm{R}^{2}=0,301 ; \\
\mathrm{R}^{2} \mathrm{aj}=0,170\end{array}$} & \multicolumn{2}{|c|}{$\begin{array}{c}\mathbf{F E} \\
R^{2}=0,356 ; \\
R^{2} a j=0,236\end{array}$} & \multicolumn{2}{|c|}{$\begin{array}{c}F C \\
R^{2}=0,308 ; \\
R^{2} a j=0,179\end{array}$} & \multicolumn{2}{|c|}{$\begin{array}{c}\text { FS } \\
\mathrm{R}^{2}=0,171 ; \\
\mathrm{R}^{2} \mathrm{aj}=0,016\end{array}$} \\
\hline & $\beta$ & $\mathrm{p}$ & $\beta$ & $p$ & $\beta$ & $p$ & $\beta$ & $p$ & $\beta$ & $p$ \\
\hline \multicolumn{11}{|l|}{ Variáveis contínuas } \\
\hline Idade & 0,02 & 0,894 & 0,23 & 0,188 & 0,25 & 0,147 & 0,04 & 0,844 & 0,00 & 0,988 \\
\hline IMC & $-0,11$ & 0,254 & 0,01 & 0,953 & $-0,39$ & $<0,001^{*}$ & $-0,16$ & 0,127 & 0,02 & 0,878 \\
\hline Apetite (escore) & $-0,43$ & $<0,001^{*}$ & $-0,23$ & 0,047 & $-0,46$ & $<0,001^{*}$ & $-0,47$ & $<0,001^{*}$ & $-0,19$ & 0,119 \\
\hline \multicolumn{11}{|l|}{ Variáveis categóricas } \\
\hline Grupo etário & $-0,04$ & 0,782 & $-0,12$ & 0,492 & $-0,15$ & 0,360 & $-0,24$ & 0,166 & $-0,11$ & 0,545 \\
\hline Sexo & $-0,03$ & 0,791 & 0,19 & 0,121 & $-0,07$ & 0,518 & $-0,16$ & 0,172 & $-0,20$ & 0,123 \\
\hline Estado civil & $-0,08$ & 0,398 & $-0,04$ & 0,734 & $-0,07$ & 0,513 & 0,10 & 0,334 & 0,11 & 0,347 \\
\hline Atividade laboral & 0,06 & 0,581 & 0,07 & 0,567 & 0,02 & 0,891 & 0,10 & 0,397 & $-0,09$ & 0,450 \\
\hline Classe econômica & 0,11 & 0,248 & $-0,11$ & 0,279 & 0,07 & 0,499 & $-0,02$ & 0,840 & 0,07 & 0,526 \\
\hline Tipo de tratamento & $-0,07$ & 0,457 & 0,00 & 0,971 & $-0,15$ & 0,135 & $-0,05$ & 0,632 & 0,13 & 0,252 \\
\hline Grupos diagnósticos & 0,09 & 0,349 & 0,20 & 0,070 & $-0,31$ & $0,004^{*}$ & $-0,14$ & 0,207 & $-0,05$ & 0,670 \\
\hline Estadiamento clínico & 0,01 & 0,960 & 158,00 & 0,201 & 0,08 & 0,490 & 0,13 & 0,290 & 0,15 & 0,261 \\
\hline Metástase & $-0,06$ & 0,593 & $-0,09$ & 0,444 & $-0,12$ & 0,292 & $-0,14$ & 0,210 & $-0,08$ & 0,519 \\
\hline ASG & $-0,07$ & 0,811 & 0,15 & 0,642 & 0,77 & $0,013^{*}$ & 0,71 & $0,026^{*}$ & $-0,11$ & 0,740 \\
\hline \multicolumn{11}{|c|}{ Escala de sintomas } \\
\hline & \multicolumn{2}{|c|}{$\begin{array}{c}\text { Fadiga } \\
\mathrm{R}^{2}=0,399 ; \\
\mathrm{R}^{2} \mathrm{aj}=0,287\end{array}$} & \multicolumn{2}{|c|}{$\begin{array}{c}\text { Náuseas/vômito } \\
R^{2}=0,427 ; \\
R^{2} a j=0,320\end{array}$} & \multicolumn{2}{|c|}{$\begin{array}{c}\text { Dor } \\
R^{2}=0,219 ; \\
R^{2} a j=0,073\end{array}$} & \multicolumn{2}{|c|}{$\begin{array}{c}\text { Dispneia } \\
\mathrm{R}^{2}=0,244 ; \\
\mathrm{R}^{2} \mathrm{aj}=0,103\end{array}$} & \multicolumn{2}{|c|}{$\begin{array}{c}\text { Insônia } \\
R^{2}=0,159 ; \\
R^{2} a j=0,002\end{array}$} \\
\hline & $\beta$ & $\mathrm{p}$ & $\beta$ & $p$ & $\beta$ & $p$ & $\beta$ & $\mathrm{p}$ & $\beta$ & $\mathrm{p}$ \\
\hline Variáveis contínuas & & & & & & & & & & \\
\hline Idade & $-0,57$ & 0,001 & $-0,30$ & 0,060 & $-0,162$ & 0,385 & $-0,26$ & 0,166 & 0,33 & 0,090 \\
\hline IMC & 0,10 & 0,293 & $-0,03$ & 0,758 & 0,012 & 0,911 & 0,11 & 0,316 & 0,16 & 0,174 \\
\hline Apetite (escore) & 0,42 & $<0,001^{*}$ & 0,306 & $0,003^{*}$ & 0,405 & $0,001^{*}$ & 0,37 & $0,002^{*}$ & 0,23 & 0,072 \\
\hline Variáveis categóricas & & & & & & & & & & \\
\hline Grupo etário & 0,31 & 0,055 & 0,05 & 0,771 & 0,047 & 0,797 & $-0,03$ & 0,889 & $-0,16$ & 0,390 \\
\hline Sexo & $-0,01$ & 0,914 & $-0,08$ & 0,460 & $-0,22$ & 0,084 & $-0,06$ & 0,637 & $-0,01$ & 0,959 \\
\hline Estado civil & 0,06 & 0,530 & $-0,01$ & 0,958 & $-0,034$ & 0,766 & 0,08 & 0,503 & $-0,12$ & 0,307 \\
\hline Atividade laboral & $-0,14$ & 0,203 & $-0,17$ & 0,111 & $-0,028$ & 0,818 & $-0,15$ & 0,212 & $-0,13$ & 0,306 \\
\hline Classe econômica & 0,08 & 0,421 & 0,24 & 0,012 & 0,052 & 0,631 & 0,10 & 0,345 & 0,06 & 0,622 \\
\hline Tipo de tratamento & 0,05 & 0,596 & 0,14 & 0,159 & $-0,031$ & 0,782 & 0,17 & 0,130 & $-0,02$ & 0,840 \\
\hline Grupos diagnósticos & 0,01 & 0,907 & $-0,05$ & 0,631 & $-0,084$ & 0,459 & $-0,06$ & 0,610 & $-0,05$ & 0,692 \\
\hline Estadiamento clínico & 0,01 & 0,935 & 0,02 & 0,895 & $-0,243$ & 0,064 & $-0,25$ & 0,053 & 0,02 & 0,892 \\
\hline Metástase & $-0,05$ & 0,617 & $-0,10$ & 0,339 & 0,148 & 0,223 & 0,07 & 0,541 & $-0,07$ & 0,591 \\
\hline ASG & $-0,06$ & 0,844 & 0,95 & $0,001^{*}$ & $-0,142$ & 0,670 & $-0,02$ & 0,953 & $-0,36$ & 0,301 \\
\hline & & & & scala de & omas & & & & & \\
\hline & & $\begin{array}{l}\text { ite } \\
506 ; \\
0,414\end{array}$ & $\begin{array}{l}\text { Con } \\
\mathrm{R}^{2} \\
\mathrm{R}^{2} \mathrm{a}\end{array}$ & $\begin{array}{l}\text { ação } \\
188 ; \\
0,037\end{array}$ & & $\begin{array}{l}\text { eia } \\
1,225 ; \\
0,081\end{array}$ & & $\begin{array}{l}214 \\
0,067\end{array}$ & $\begin{array}{r}\text { Saúde } \\
R^{2} \\
R^{2}\end{array}$ & $\begin{array}{l}\mathrm{bal} / Q \mathrm{QV} \\
436 ; \\
0,330\end{array}$ \\
\hline & $\beta$ & $p$ & $\beta$ & $p$ & $\beta$ & p & $\beta$ & p & $\beta$ & $p$ \\
\hline Variáveis contínuas & & & & & & & & & & \\
\hline Idade & $-0,17$ & 0,242 & 0,04 & 0,822 & $-0,15$ & 0,419 & 0,28 & 0,144 & 0,25 & 0,118 \\
\hline IMC & 0,02 & 0,789 & 0,27 & $0,019 *$ & $-0,07$ & 0,555 & $-0,10$ & 0,364 & $-0,11$ & 0,270 \\
\hline Apetite (escore) & 0,67 & $<0,001^{*}$ & 0,10 & 0,405 & 0,09 & 0,427 & $-0,24$ & $0,049^{*}$ & $-0,54$ & $<0,001^{*}$ \\
\hline Variáveis categóricas & & & & & & & & & & \\
\hline Grupo etário & 0,02 & 0,907 & $-0,31$ & 0,103 & $-0,13$ & 0,482 & $-0,11$ & 0,533 & $-0,12$ & 0,426 \\
\hline Sexo & $-0,18$ & 0,070 & $-0,01$ & 0,960 & 0,03 & 0,825 & 0,13 & 0,295 & 0,21 & 0,056 \\
\hline Estado civil & 0,04 & 0,687 & 0,04 & 0,707 & $-0,09$ & 0,405 & 0,11 & 0,328 & $-0,04$ & 0,676 \\
\hline Atividade laboral & $-0,01$ & 0,928 & $-0,05$ & 0,660 & $-0,27$ & $0,029^{*}$ & $-0,05$ & 0,668 & $-0,07$ & 0,505 \\
\hline Classe econômica & $-0,01$ & 0,958 & 0,12 & 0,283 & $-0,19$ & 0,084 & $-0,19$ & 0,081 & $-0,17$ & 0,077 \\
\hline Tipo de tratamento & 0,08 & 0,349 & $-0,04$ & 0,704 & $-0,12$ & 0,279 & $-0,06$ & 0,604 & 0,02 & 0,803 \\
\hline Grupos diagnósticos & $-0,06$ & 0,508 & 0,05 & 0,681 & $-0,08$ & 0,469 & $-0,02$ & 0,843 & $-0,07$ & 0,491 \\
\hline Estadiamento clínico & $-0,01$ & 0,905 & $-0,10$ & 0,471 & 0,26 & 0,050 & 0,19 & 0,158 & 0,02 & 0,825 \\
\hline Metástase & $-0,08$ & 0,386 & 0,07 & 0,586 & $-0,07$ & 0,558 & $-0,02$ & 0,901 & $-0,15$ & 0,148 \\
\hline ASG & $-0,47$ & 0,079 & $-0,37$ & 0,275 & $-0,21$ & 0,535 & $-0,52$ & 0,125 & 0,11 & 0,698 \\
\hline
\end{tabular}

Legendas: EORTC/QLQ C-30: European Organization for Research Treatment of Cancer Quality of Life Questionnaire Core 30; FF: funçăo física; DP: desempenho de papel; FE: função emocional; FC: função cognitiva; FS: função social; DF: dificuldade financeira; QV: qualidade de vida; IMC: índice de massa corporal; ASG: avaliação subjetiva global. 
prognóstico, de forma que minimize maiores complicaçóes ao longo do tratamento. Dessa forma, os achados deste estudo confirmam que a ASG é uma ferramenta essencial para identificar precocemente o risco nutricional de pacientes submetidos aos tratamentos antioneoplásicos.

Além disso, investigar o comprometimento do apetite e a presença de sintomas de impacto nutricional é fundamental em todas as fases do tratamento do câncer, independentemente do tipo de tumor, pois alteraçôes do apetite são comuns nos pacientes com câncer e impactam na QVRS dos pacientes ${ }^{17}$.

Outro aspecto relevante encontrado neste estudo foi a $\mathrm{FE}$ ter sido a mais prejudicada entre os pacientes. Isso se deve, provavelmente, às mudanças que ocorrem na vida do indivíduo com câncer após a notícia do diagnóstico e relacionadas ao próprio tratamento clínico, especialmente o quimioterápico, pois pode afetar diretamente o aspecto emocional, por se tratar de um processo, na maioria das vezes, longo, cansativo e doloroso ${ }^{16}$. Estudo conduzido por Lobo et al. ${ }^{18}$, no qual avaliaram a QV de 145 mulheres com neoplasias de mama em tratamento quimioterápico, encontrou que a FE também foi a função que apresentou escore médio mais baixo $(61,32)$, o que corrobora os nossos achados.

Neste estudo, a função com média mais elevada foi o DP. Essa função é composta pelos itens 6 ("Você apresentou limitaçóes para realizar seu trabalho ou fazer outras atividades diárias?") e 7 ("Você apresentou limitaçóes para realizar atividades de divertimento ou lazer?") do instrumento. Tal fato pode ter ocorrido em virtude de a população estudada ter sido entrevistada em regime ambulatorial e encontrar-se com bom estado nutricional segundo ASG. Esses aspectos podem ter sido importantes para a maioria dos pacientes que relatou não apresentar dificuldade para realizar atividades de trabalho, diárias ou até mesmo de lazer. Outro aspecto a ser considerado é que, embora a prevalência neste estudo seja de idosos, grande parte destes não deixou suas atividades de trabalho por motivo da doença e/ou tratamento antineoplásico.

A fadiga é um sintoma muito comum e frequentemente reportado por pacientes oncológicos em diversas fases da doença e/ou tratamento ${ }^{16,17}$, especialmente por aqueles que apresentam a doença em estágios mais avançados e em tratamento quimioterápico. Geralmente, os pacientes apresentam sensação persistente de cansaço, inclusive a mínimos esforços ${ }^{3}$. A escala de fadiga foi a que apresentou pontuaçáo mais elevada, sendo, portanto, a sintomatologia de destaque no presente estudo. Cabe ressaltar que a maioria dos pacientes entrevistados se encontra em estágios III e IV da doença $(64,44 \%)$, sendo a fadiga uma característica importante do câncer avançado e dos pacientes submetidos ao tratamento quimioterápico $(\mathrm{n}=88,80,0 \%)$. A fadiga relacionada ao câncer é muito comum, em razão das comorbidades à que esses pacientes sáo sujeitos ${ }^{19}$. Estudo de coorte prospectivo, dirigido por Pearce et al. ${ }^{20}$, realizado com 449 indivíduos acompanhados por 5,64 meses, encontrou que $27 \%$ dos pacientes relataram apresentar fadiga como efeito colateral do tratamento, sendo $85 \%$ dos casos, confirmando com os resultados encontrados no presente estudo.

No que tange ao item isolado DF, o escore médio foi elevado nesses pacientes, o que significa que a condiçáo física e/ou tratamento médico causaram dificuldades financeiras. Mesmo em se tratando de um estudo conduzido em uma clínica privada, que presta atendimento a pacientes que possuem convênios médicos de saúde, a doença e o tratamento impactam na vida financeira do paciente.

Cabe esclarecer algumas limitaçôes deste trabalho, como o estudo ter sido tipo transversal; a náo homogeneidade entre os diagnósticos e os tipos de tratamento; e o fato de ser uma população extremamente heterogênea em relação à topografia. Sendo assim, incentiva-se que novos estudos sejam conduzidos com intuito de dar continuidade às investigaçóes sobre QVRS, alteraçóes do apetite e estado nutricional de pacientes com câncer. Como pontos fortes, podem ser considerados a iniciativa de conduzir estudos com essa população no Centro-Oeste do Brasil e a contribuição dos nossos achados para a prática clínica do nutricionista e de profissionais da área da saúde.

\section{CONCLUSÃO}

O comprometimento do apetite foi associado à saúde global e à QV dos pacientes com câncer em tratamento clínico. Embora para esta amostra o estado nutricional avaliado pela ASG não tenha apresentado associação no modelo de regressão linear, cujo desfecho foi a saúde geral/QV, é um aspecto a ser considerado nos modelos de QVRS, uma vez que apresentou associaçáo significativa com a FE, FC e náuseas e vômitos.

As características sociodemográficas e clínicas não apresentaram relação com QVRS dos pacientes que compuseram este estudo e/ou não se mostraram relevantes nesta amostra. Mais da metade dos pacientes relataram algum grau de fadiga, sendo essa condição a de pontuação mais elevada, seguida por insônia e falta de apetite. Embora a saúde geral e a QVRS dos pacientes estejam relativamente preservadas, a FE dos pacientes foi a mais acometida.

\section{CONTRIBUIÇÕES}

Todas as autoras participaram de todas as etapas do manuscrito e aprovação da versão final. 


\section{DECLARAÇÃO DE CONFLITO DE INTERESSES}

Nada a declarar.

\section{FONTES DE FINANCIAMENTO}

Não há

\section{REFERÊNCIAS}

1. Organização das Nações Unidas (BR). Casos de câncer devem aumentar $70 \%$ até 2038 , calcula OMS [Internet]. Brasília, DF: ONUBR; 2018 fev. 5. [acesso 2019 jul.15]. Disponível em: https://nacoesunidas.org/casos-decancer-devem-aumentar-70-ate-2038-calcula-oms/

2. Instituto Nacional de Câncer José Alencar Gomes da Silva. Estima 2018: incidência de câncer no Brasil [Internet]. Rio de Janeiro: INCA; 2017 [acesso 2019 jul. 15]. Disponível em: http://www1.inca.gov.br/ estimativa/2018/

3. Guimarães RM, Sousa ALC, Oliveira CM, et al. Avaliação nutricional e da qualidade de vida de pacientes com câncer do aparelho digestório. Saúde Rev. 2016;16(44):6374. doi: http://dx.doi.org/10.15600/2238-1244/ sr.v16n44p63-74

4. Aaronson NK, Ahmedzai S, Bergman B, et al. The European Organization for Research and Treatment of Cancer QLQ-C30: a quality-of-life instrument for use in international clinical trials in oncology. J Natl Cancer Inst. 1993;85(5):365-76. doi: http://dx.doi.org/10.1093/ jnci/85.5.365

5. Campos JADB, Spexoto MCB, Silva WR, et al. European Organization for Research and Treatment of Cancer Quality of Life questionnaire core 30: factorial models to Brazilian cancer patients. Einstein (Sáo Paulo). 2018;16(1):eAO4132. http://dx.doi.org/10.1590/ s1679-45082018ao4132

6. Soares LC, Burille A, Antonacci MH, et al. A quimioterapia e seus efeitos adversos: relato de clientes oncológicos. Cogitare Enferm. 2009;14(4):714-9. http:// dx.doi.org/10.5380/ce.v14i4.16388

7. Poltronieri TS, Tusset C. Impacto do tratamento do câncer sobre o estado nutricional de pacientes oncológicos: atualização da literatura. Rev Bras Cienc Saúde. 2016;20(4):327-32.

8. Associaçấo Brasileira de Empresas de Pesquisa. Critério de classificação econômica Brasil [Internet]. São Paulo: ABEP; 2018 [acesso 2019 jul. 15]. Disponível em: http:// www.abep.org/criterio-brasil

9. Halliday V, Porock D, Arthur A, et al. Development and testing of a cancer appetite and symptom questionnaire. J Hum Nutr Diet. 2012;25(3):217-24. doi: https://doi. org/10.1111/j.1365-277X.2012.01233.x
10. Spexoto MC, Serrano SV, Halliday V, et al. Cancer Appetite and Symptom Questionnaire (CASQ) for Brazilian patients: cross-cultural adaptation and validation study. PLoS One. 2016;11(6):e0156288. doi: https://doi.org/10.1371/journal.pone.0156288

11. Ottery FD. Definition of standardized nutritional assessment and interventional pathways in oncology. Nutrition. 1996;12(1 Suppl):S15-9. doi: https://doi. org/10.1016/0899-9007(96)90011-8

12. Campos JADB, Prado CD. Cross-cultural adaptation of the Portuguese version of the patient-generated subjective global assessment. Nutr Hosp. 2012;27(2):583-9. doi: https://doi.org/10.1590/S0212-16112012000200035

13. World Health Organization. Obesity: preventing and managing the global epidemic: report of a WHO Consultation [Internet]. Geneva: WHO; 2000 [cited 15 Jul 2019]. (WHO technical report series; 894). Available from: https://www.who.int/nutrition/publications/ obesity/WHO_TRS_894/en/

14. Lipschitz DA. Screening for nutritional status in the elderly. Prim Care. 1994;21(1):55-67.

15. Jager-Wittenaar H, Ottery FD. Assessing nutritional status in cancer: Role of the Patient-Generated Subjective Global Assessment. Curr Opin Clin Nutr Metab Care. 2017;20(5):322-29. doi: https://doi.org/10.1097/ MCO.0000000000000389

16. Sociedade Brasileira de Nutrição Parenteral e Enteral. Diretriz BRASPEN de terapia nutricional no paciente com câncer. BRASPEN J. 2019;34(Supl 1):2-32.

17. Campos JADB, Silva WR, Spexoto MCB, et al. Características clinicas, dietéticas e demográficas que interferem na qualidade de vida de pacientes com câncer. Einstein (Sáo Paulo). 2018;16(4):eAO4368. doi: https:// doi.org/10.31744/einstein_journal/2018AO4368

18. Lobo SA, Fernandes AFC, Almeida PC, et al. Qualidade de vida em mulheres com neoplasias de mama em quimioterapia. Acta Paul Enferm. 2014;27(6):554-9. doi: http://dx.doi.org/10.1590/1982-0194201400090

19. Arends J, Bachmann P, Baracos V, et al. ESPEN guidelines on nutrition in cancer patients. Clin Nutr. 2017;36(1):11-48. doi: https://doi.org/10.1016/j. clnu.2016.07.015

20. Pearce A, Haas M, Viney R, et al. Incidence and severity of self-reported chemotherapy side effects in routine care: a prospective cohort study. Plos One. 2017;12(10): e0184360. doi: https://doi.org/10.1371/journal. pone. 0184360

Recebido em 28/8/19 Aprovado em 24/10/19 